\title{
Plasmatic Coagulation Capacity Correlates With Inflammation and Abacavir Use During Chronic HIV Infection
}

Citation for published version (APA):

van der Heijden, W. A., Wan, J., Van de Wijer, L., Jaeger, M., Netea, M. G., van der Ven, A. J., de Groot, P. G., Roest, M., \& de Mast, Q. (2021). Plasmatic Coagulation Capacity Correlates With Inflammation and Abacavir Use During Chronic HIV Infection. Jaids-journal of Acquired Immune Deficiency Syndromes, 87(1), 711-719. https://doi.org/10.1097/qai.0000000000002633

Document status and date:

Published: 01/05/2021

DOI:

10.1097/qai.0000000000002633

Document Version:

Publisher's PDF, also known as Version of record

Document license:

Taverne

Please check the document version of this publication:

- A submitted manuscript is the version of the article upon submission and before peer-review. There can be important differences between the submitted version and the official published version of record.

People interested in the research are advised to contact the author for the final version of the publication, or visit the DOI to the publisher's website.

- The final author version and the galley proof are versions of the publication after peer review.

- The final published version features the final layout of the paper including the volume, issue and page numbers.

Link to publication

\footnotetext{
General rights rights.

- You may freely distribute the URL identifying the publication in the public portal. please follow below link for the End User Agreement:

www.umlib.nl/taverne-license

Take down policy

If you believe that this document breaches copyright please contact us at:

repository@maastrichtuniversity.nl

providing details and we will investigate your claim.
}

Copyright and moral rights for the publications made accessible in the public portal are retained by the authors and/or other copyright owners and it is a condition of accessing publications that users recognise and abide by the legal requirements associated with these

- Users may download and print one copy of any publication from the public portal for the purpose of private study or research.

- You may not further distribute the material or use it for any profit-making activity or commercial gain

If the publication is distributed under the terms of Article $25 \mathrm{fa}$ of the Dutch Copyright Act, indicated by the "Taverne" license above, 


\title{
Plasmatic Coagulation Capacity Correlates With Inflammation and Abacavir Use During Chronic HIV Infection
}

\author{
Wouter A. van der Heijden, MD, ${ }^{a}$ Jun Wan, MSc, ${ }^{b}$ Lisa Van de Wijer, MD, ${ }^{a}$ Martin Jaeger, PhD, ${ }^{a}$ \\ Mihai G. Netea, MD, PhD, ${ }^{a, c}$ Andre J. van der Ven, MD, PhD, ${ }^{a}$ Philip G. de Groot, PhD, ${ }^{b}$ \\ Mark Roest, PhD, ${ }^{b}$ and Quirijn de Mast, MD, PhD ${ }^{a}$
}

\begin{abstract}
Background: D-dimer concentrations in people living with HIV (PLHIV) on combination antiretroviral therapy (cART) are increased and have been linked to mortality. D-dimer is a biomarker of in vivo coagulation. In contrast to reports on D-dimer, data on coagulation capacity in PLHIV are conflicting. In this study, we assessed the effect of cART and inflammation on coagulation capacity.
\end{abstract}

Setting: We explored coagulation capacity using calibrated thrombin generation (TG) and linked this to persistent inflammation and cART in a cross-sectional study including PLHIV with viral suppression and uninfected controls.

Methods: We used multivariate analyses to identify independent factors influencing in vivo coagulation (D-dimer) and ex vivo coagulation capacity (TG).

Results: Among 208 PLHIV, 94 (45\%) were on an abacavircontaining regimen. D-dimer levels $(219.1$ vs $170.5 \mathrm{ng} / \mathrm{mL}, P=$

Received for publication October 7, 2020; accepted December 21, 2020.

From the a Department of Internal Medicine, Radboud Center for Infectious Diseases, Radboud University Medical Center, Nijmegen, the Netherlands; 'bynapse Research Institute, Cardiovascular Research Institute Maastricht, Maastricht University Medical Center, Maastricht, the Netherlands; and ${ }^{c}$ Department for Genomics and Immunoregulation, Life and Medical Sciences 12 Institute (LIMES), University of Bonn, Bonn, Germany.

Supported in part by an AIDS-fonds Netherlands Grant. M.G.N. was supported by a Spinoza grant of the Netherlands Organization for Scientific Research and an ERC Advanced Grant (\#833247). J.W. was supported by a scholarship (No. 201606130068) from the China Scholarship Council. All contributors were not involved in the study design, data interpretation, or the submission.

M.R. is employed by Synapse Research Institute, which is a member of the Stago group. The other authors have no funding or conflicts of interest to disclose.

Supplemental digital content is available for this article. Direct URL citations appear in the printed text and are provided in the HTML and PDF versions of this article on the journal's Web site (www.jaids.com).

W.A.H, Q.M., M.R., and A.J.V. designed the study. W.A.H., L.V.W., and M.J. recruited and included the participants. W.A.H., J.W., and L.W. performed the laboratory experiments. W.A.H. and J.W. analyzed the data and interpreted the data together with Q.M., P.G.G., M.R., and A.J.V. W.A.H., J.W., A.J.V., and Q.M. wrote the manuscript. All authors have read and contributed significantly to the final manuscript.

Correspondence to: Wouter A. van der Heijden, MD, Department of Internal Medicine, Radboud University Medical Center, 6500 HB Nijmegen, Netherlands (e-mail: Wouter.vanderHeijden@radboudumc.nl).

Copyright (C) 2021 Wolters Kluwer Health, Inc. All rights reserved.
$0.001)$ and inflammatory makers (sCD14, sCD163, and highsensitive C-reactive protein) were increased in PLHIV compared with those in controls $(\mathrm{n}=56)$. PLHIV experienced lower TG (reflected by endogenous thrombin potential [ETP]) when compared with controls, after correction for age, sex, and antiretroviral therapy. Abacavir use was independently associated with increased ETP. Prothrombin concentrations were strongly associated with ETP and lower in PLHIV on a non-abacavir-containing regimen compared with those in controls, suggesting consumption as a possible mechanism for HIV-associated reduction in TG. D-dimer concentrations were associated with inflammation, but not TG.

Conclusions: Abacavir use was associated with increased TG and could serve as an additional factor in the reported increase in thrombotic events during abacavir use. Increased exposure to triggers that propagate coagulation, such as inflammation, likely underlie increased D-dimer concentrations found in most PLHIV.

Key Words: D-dimer, thrombin generation, abacavir, inflammation, coagulation, thrombosis

(J Acquir Immune Defic Syndr 2021;87:711-719)

\section{INTRODUCTION}

Successful combination antiretroviral therapy (cART) has nearly normalized life expectancy of people living with HIV (PLHIV), although treatment is not able to fully reverse immune activation and persistent inflammation. ${ }^{1-3}$ This inflammation may increase the risk of thrombotic events. ${ }^{4}$ Although increased risk of venous thrombosis in PLHIV seems to be limited to those with incomplete CD4 recovery or with continuing viral replication, ${ }^{5,6}$ data on arterial thrombosis risk show an increased cardiovascular risk in well-treated PLHIV. ${ }^{7-9}$ There is an ongoing debate on the effect of cART, especially abacavir, on cardiovascular risk. ${ }^{10-15}$

D-dimers, which are soluble fibrin degradation products, are markers of coagulation activity in the body. HIV infection is associated with elevated D-dimer concentrations even in those with viral suppression. ${ }^{16-18}$ D-dimer concentrations are independently associated with overall mortality and the incidence of cardiovascular disease (CVD) and cancer in PLHIV. ${ }^{9,16,19}$ D-dimer concentrations are influenced by 
activation of the coagulation cascade itself and signals that provoke coagulation such as inflammation and endothelial activation. ${ }^{4}$ Indeed, the inflammatory markers sCD14, highsensitive C-reactive protein (hsCRP), and sCD163 have been associated with D-dimer in PLHIV ${ }^{17,20}$ suggesting a link between inflammation and in vivo coagulation activity in PLHIV.

The capacity of plasma to form thrombin is a critical determinant of in vivo plasmatic coagulation. ${ }^{21}$ Thrombin generation (TG) can be measured ex vivo to determine coagulation capacity in a standardized setting and has been used as a diagnostic tool for hypocoagulability and hypercoagulability states. ${ }^{22}$ In contrast to elevated D-dimer concentrations in PLHIV, ${ }^{16-18}$ available data on ex vivo $\mathrm{TG}$ in PLHIV are contradictory. ${ }^{23-25}$ This also applies to the possible roles of inflammation and cART on TG.

Hence, we measured TG and different coagulation markers in a cross-sectional cohort of cART treated, virally suppressed PLHIV and HIV-uninfected controls and related TG to markers of inflammation and cART. We hypothesized that TG is influenced by both persistent inflammation and cART.

\section{MATERIALS AND METHODS}

\section{Study Procedures}

This cross-sectional, prospective study was performed at the Radboud University Medical Center, a tertiary teaching hospital in the Netherlands. The study was conducted in accordance with the Declaration of Helsinki after approval of the ethics committee (CMO Arnhem-Nijmegen, the Netherlands; NL42561.091.12, 2012/550). This study was embedded in the Human Functional Genomics Project (HFGP; www. humanfunctionalgenomicsproject.org). Adult HIV-1-infected individuals and controls were concurrently enrolled after written informed consent was obtained. Inclusion criteria included suppressed viral load ( $<200$ copies $/ \mathrm{mL}$ ) after cART use for at least 6 months. Exclusion criteria were the use of coumarin derivates or direct anticoagulant therapy, active hepatitis $\mathrm{B}$ or $\mathrm{C}$ infection, and/or signs of an infection other than HIV-1.

\section{Plasma TG Measured With and Without Thrombomodulin}

TG in platelet poor plasma (PPP) was measured with the "MIDIcat" technique, which is a modified calibrated TG [Calibrated Automated Thrombogram (CAT)] for the measurement of samples with low plasma volume. ${ }^{22,26}$ This technique maintains the plasma dilution ratio, whereas requiring only half of the volume (ie, 40 versus $80 \mu \mathrm{L}$ per well) needed than the regular CAT technique. PPP was stored at $-80^{\circ} \mathrm{C}$ until thawed at $37^{\circ} \mathrm{C}$ for 10 minutes before measurement. TG was triggered with $5 \mathrm{pM}$ tissue factor (TF; Innovin; Siemens Healthcare Diagnostics, Marburg, Germany), $4 \mu \mathrm{M}$ phospholipids (PL; Avanti Polar Lipids Inc., Alabaster, AL), and in the presence and absence of $7 \mathrm{nM}$ thrombomodulin (TM; Synapse Research Institute, Maastricht, the Netherlands). The concentration of TM was chosen to inhibit the endogenous thrombin potential (ETP) by $50 \%$ in normal pooled plasma (NPP). TG parameters were calculated using specialized software from Thrombinoscope B.V. (Maastricht, the Netherlands). TG parameters (see Table, Supplemental Digital Content, http://links.lww.com/QAI/ B606) including lag time (LT; minutes), time-to-peak (TTP; minutes), peak (nM), ETP $(\mathrm{nM} \times$ minutes $)$, and velocity index (VI; $\mathrm{nM} / \mathrm{min}$ ) were chosen for further analysis. The ETP, peak, and VI of tested subjects were normalized as the percentage of that of NPP tested without TM in the same run. ${ }^{26}$

The sensitivity of the TG parameters to TM reflects the function of the anticoagulant protein $C$ pathway. The TM sensitivity ratio of ETP (ETP-TMsr) was calculated as the ratio of ETP in the presence of TM and ETP in the absence of TM. The normalized TM sensitivity ratio of ETP (nETPTMsr) was calculated by dividing the ETP-TMsr of subject by that of NPP in the same run. The nPeak-TMsr and nVITMsr were calculated similarly. An nTMsr value less than 1 means that the PPP of the tested subject has a better functioning protein $\mathrm{C}$ system than that of the NPP and vice versa.

\section{Plasma Markers of Coagulation and Inflammation}

Inflammatory markers sCD163 (Quantikine), sCD14 (Quantikine), and hsCRP (Duoset) were determined in EDTA plasma by enzyme-linked immunosorbent assay (ELISA; all R\&D system, Minneapolis, MI). D-dimer was measured by ELISA according to the manufacturer's instructions (Abcam, Cambridge, United Kingdom).

The measurement of plasmatic fibrinogen was performed using the Clauss method on a STart4 analyzer (Diagnostica Stago, Asnières, France) with a known fibrinogen reagent (Dade Fibrinogen Determination Reagent, Siemens, Munich, Germany). Prothrombin (sheep antihuman prothrombin polyclonal antibody and horseradish peroxidase (HRP)-conjugated sheep antihuman prothrombin polyclonal antibody; Affinity Biologicals Inc., Ancaster, Canada), protein $\mathrm{S}$ (sheep antihuman protein S) IgG antibody and HRPconjugated sheep antihuman protein $\mathrm{S}$ antibody from Affinity Biologicals, Inc., and von Willebrand factor (vWF; rabbit antihuman VWF and HRP-conjugated rabbit antihuman vWF; DAKO, Agilent, Santa Clara, CA) concentrations were performed with an in-house sandwich ELISA assay. In brief, 96-well microtiter plates (NUNC MaxiSorp; Thermo Fisher Scientific, Waltham, MA) were coated overnight at $4^{\circ} \mathrm{C}$ with capture antibody in a carbonate-bicarbonate coating buffer ( $\mathrm{pH} 9.6$ ) and blocked with $2 \%$ bovine serum albumin (BSA) in phosphate-buffered saline (PBS) for 45 minutes at room temperature (RT) before adding diluted plasma samples and incubated at RT for 1.5 hours. The wells were then incubated with detection antibody in $\mathrm{PBS} / 2 \% \mathrm{BSA}$ for 2 hours at room temperature after washing. Plates were washed before addition of SIGMAFAST OPD (Sigma). After 30 minutes, the reaction was stopped with $3 \mathrm{M}$ sulfuric acid $\left(\mathrm{H}_{2} \mathrm{SO}_{4}\right.$; Sigma). Optical densities (ODs) were measured at $490 \mathrm{~nm}$ using an ELx808 Absorbance Microplate Reader (Biotek, Bad Friedrichshall, Germany). A calibration curve of serial 
TABLE 1. Baseline Characteristics of PLWHIV and Uninfected Controls Stratified by Abacavir Use

\begin{tabular}{|c|c|c|c|c|c|}
\hline & No $\mathbf{A B C}$ & $\mathbf{A B C}$ & HC & ABC vs No ABC & HC vs PLHIV \\
\hline $\mathrm{N}$ & 114 & 94 & 56 & & \\
\hline Sex (female) $(\%)$ & $13(11.4)$ & $5(5.3)$ & $\begin{array}{l}22 \\
(39.3)\end{array}$ & 0.192 & $<0.001$ \\
\hline Age [median (IQR)] & $53.0(47.0,60.0)$ & $50.0(41.5,58.0)$ & $30.0(25.8,53.0)$ & 0.086 & $<0.001$ \\
\hline BMI [median (IQR)] & $24.2(22.4,26.0)$ & $23.8(21.8,26.2)$ & $23.8(21.5,25.6)$ & 0.469 & 0.518 \\
\hline HIV infection [median (IQR)], yr & $10.0(6.2,16.8)$ & $6.5(4.3,10.3)$ & & $<0.001$ & \\
\hline Way of transmission $(\%)$ & & & & 0.245 & \\
\hline Heterosexual & $3(2.6)$ & $5(5.3)$ & & & \\
\hline IDU & $2(1.8)$ & $1(1.1)$ & & & \\
\hline MSM & $82(71.9)$ & $75(79.8)$ & & & \\
\hline Other/unknown & $27(23.7)$ & $13(13.8)$ & & & \\
\hline CD4 nadir [median (IQR)] & $\begin{array}{c}205.0 \\
(120.0,347.5)\end{array}$ & $\begin{array}{c}275.0 \\
(185.0,377.5)\end{array}$ & & 0.044 & \\
\hline CD4 count [median (IQR)] & $645.0(482.5,827.5)$ & $665.0(492.5,800.0)$ & & 0.754 & \\
\hline Viral load $<40$ copies $/ \mathrm{mL}$ & $111(97.3)$ & $94(100 \%)$ & & 0.317 & \\
\hline CD4-CD8 ratio & $0.8(0.5,1.1)$ & $0.8(0.6,1.1)$ & & 0.954 & \\
\hline cART duration & $7.9(4.9,15.4)$ & $5.5(3.7,8.6)$ & & $<0.001$ & \\
\hline NNRT (\%) & $44(38.6)$ & $17(18.1)$ & & 0.002 & \\
\hline PI $(\%)$ & $26(22.8)$ & $6(6.4)$ & & 0.002 & \\
\hline INSTI $(\%)$ & $67(58.8)$ & $73(77.7)$ & & 0.006 & \\
\hline NRTI FTC (\%) & $91(79.8)$ & $0(0.0)$ & & $<0.001$ & \\
\hline NRTI 3 TC (\%) & $14(12.3)$ & $91(96.8)$ & & $<0.001$ & \\
\hline NRTI AZT (\%) & $3(2.6)$ & $1(1.1)$ & & 0.755 & \\
\hline NtRTI TDF (\%) & $92(80.7)$ & $1(1.1)$ & & $<0.001$ & \\
\hline Previous myocardial infarction (\%) & $6(5.3)$ & $4(4.3)$ & & 0.99 & \\
\hline Previous stroke $(\%)$ & $2(1.8)$ & $1(1.1)$ & & 1 & \\
\hline VTE or pulmonary embolism (\%) & $3(2.6)$ & $1(1.1)$ & & 0.755 & \\
\hline Smoking $(\%)$ & $33(28.9)$ & $24(25.5)$ & & 0.694 & \\
\hline Hypercholesterolemia (\%) & $33(28.9)$ & $23(24.5)$ & & 0.57 & \\
\hline Hypertension $(\%)$ & $23(20.2)$ & $15(16.0)$ & & 0.546 & \\
\hline Diabetes mellitus (\%) & $6(5.3)$ & $2(2.1)$ & & 0.419 & \\
\hline Family history of CVD first degree (\%) & $59(51.8)$ & $44(46.8)$ & & 0.568 & \\
\hline Cholesterol-lowering drugs $(\%)$ & $35(30.7)$ & $21(22.3)$ & & 0.232 & \\
\hline Antihypertensive drugs (\%) & $27(23.7)$ & $20(21.3)$ & & 0.805 & \\
\hline ASA $(\%)$ & $10(8.8)$ & $8(8.5)$ & & 1 & \\
\hline
\end{tabular}

Data were analyzed using either $\chi^{2}$ test or Mann-Whitney $U$ test depending on data distribution.

3TC, lamivudine; ABC, abacavir; ASA, acetyl salicylic acid; AZT, zidovudine; FTC, emtricitabine; IDU, intravenous drug use; HIV-RNA blib, viral load of 40-500 copies/mL after previous viral suppression and direct subsequent suppressed viral load; MSM, men who have sex with men; NtRTI, nucleotide reverse transcriptase inhibitor.

diluted NPP was added to each plate. ${ }^{27,28}$ Consequently, the concentrations of prothrombin and protein $\mathrm{S}$ were expressed as the percentage of the normal NPP.

\section{Statistical Analysis}

$\mathrm{R}$ version 3.5.1 (CRAN-project) was used for analyses. Comparison between groups was performed by Mann-Whitney $U$ test, Student $t$ test, or $\chi^{2}$ test depending on data distribution. The primary outcome for TG used in our analyses was ETP. Other TG parameters were treated as exploratory parameters. The Benjamini-Hochberg procedure was performed on circulating markers and ETP comparisons (Fig. 2, Table 2) to correct for the false discovery rate (FDR). For univariate and multivariate linear regression, data of the dependent variable were transformed by log- or inverse rank transformation depending on distribution. All multivariate linear regression models include a parameter to correct for possible storage degradation or time of inclusion bias. The correlation matrix was performed using Spearman correlation coefficient. Missing data for all parameters were $<2.5 \%$, and comparisons were performed pairwise.

\section{RESULTS}

A total of 208 virally suppressed PLHIV on stable cART and 56 uninfected controls were concurrently measured and included in the analysis. Baseline characteristics can be found in Table 1. PLHIV were more often male subjects (91.3\% vs $60.7 \%, P<0.001)$ and older [52 (46-59) vs 30 (26-53) years, $P<0.001]$ when compared with HIVuninfected controls. An abacavir-containing regimen was used by 94 PLHIV (45\%), and 140 PLHIV (67\%) used an integrase inhibitor (INSTI)-based regimen.

First, in vivo coagulation activity, determined by D-dimer, was increased in PLHIV compared with that in controls (219.1 vs $170.5 \mathrm{ng} / \mathrm{mL}$, respectively, $P=0.001$ ) (Table 2), as were all markers of inflammation (hsCRP, 


\begin{tabular}{|c|c|c|c|c|c|c|}
\hline & PLHIV & $\mathrm{HC}$ & PLHIV vs HC & PLHIV—No ABC & PLHIV-ABC & Non-ABC vs $\mathbf{A B C}$ \\
\hline $\mathrm{N}$ & 208 & 56 & $P$ & 114 & 94 & $P$ \\
\hline D-dimer & $219.1(160.6,334.8)$ & $170.5(122.0,307.2)$ & $0.002 *$ & $213.4(158.6,335.5)$ & $221.9(162.6,334.2)$ & 0.673 \\
\hline Fibrinogen & $3.4(2.8,4.0)$ & $3.2(2.8,4.1)$ & 0.746 & $3.4(2.8,4.0)$ & $3.3(2.8,4.0)$ & 0.632 \\
\hline Prothrombin & $109.0(89.3,135.1)$ & $135.1(102.4,162.5)$ & $<0.001 *$ & $106.0(87.1,135.1)$ & $113.4(94.8,134.8)$ & 0.305 \\
\hline Protein S & $91.4(80.7,110.3)$ & $86.9(72.7,112.4)$ & 0.121 & $91.7(81.7,109.4)$ & $91.4(78.2,110.4)$ & 0.937 \\
\hline hsCRP & $1446(608,2735)$ & $651(205,1179)$ & $<0.001^{*}$ & $1558(624,3285)$ & $1192(594,2249)$ & 0.337 \\
\hline sCD14 & $2139(1778,2625)$ & $1789(1502,2071)$ & $<0.001 *$ & $2063(1745,2591)$ & $2236(1830,2684)$ & 0.193 \\
\hline sCD163 & $716.2(525.6,898.8)$ & $517.3(410.7,578.1)$ & $<0.001 *$ & $765.8(581.6,916.1)$ & $634.5(487.8,858.3)$ & 0.019 \\
\hline ETP & $86.6(78.6,99.6)$ & $93.0(83.3,105.0)$ & $0.011^{*}$ & $83.7(74.7,97.6)$ & $90.2(82.7,101.6)$ & $0.001 *$ \\
\hline LT & $2.0(1.7,2.3)$ & $2.0(1.7,2.0)$ & 0.382 & $2.0(1.7,2.3)$ & $2.0(1.7,2.3)$ & 0.557 \\
\hline TTP & $4.4(4.0,5.0)$ & $4.3(3.5,5.0)$ & 0.035 & $4.3(4.0,5.0)$ & $4.4(4.0,5.0)$ & 0.566 \\
\hline LagT-TM & $1.7(1.4,2.0)$ & $1.7(1.4,2.0)$ & 0.704 & $1.7(1.4,2.3)$ & $1.7(1.7,2.0)$ & 0.645 \\
\hline TTP-TM & $3.7(3.3,4.0)$ & $3.7(3.3,4.0)$ & 0.641 & $3.7(3.3,4.0)$ & $3.7(3.3,4.0)$ & 0.982 \\
\hline ЕТР-ТМ & $40.6(27.9,52.0)$ & $50.1(32.8,76.2)$ & $<0.001$ & $41.0(28.0,51.1)$ & $39.7(26.3,52.6)$ & 0.706 \\
\hline PEAK-TM & $49.6(31.8,62.4)$ & $57.1(39.8,82.5)$ & 0.002 & $50.9(33.2,61.6)$ & $47.9(29.9,62.5)$ & 0.553 \\
\hline VI-TM & $59.8(38.6,82.5)$ & $71.9(51.8,112.5)$ & $<0.001$ & $62.1(39.8,85.4)$ & $59.6(33.2,77.5)$ & 0.509 \\
\hline nETP-TMSR & $0.9(0.7,1.2)$ & $1.2(0.8,1.5)$ & 0.001 & $0.9(0.7,1.2)$ & $0.9(0.6,1.2)$ & 0.183 \\
\hline nPeak-TMSR & $0.9(0.8,1.1)$ & $1.1(0.9,1.3)$ & $<0.001$ & $1.0(0.8,1.1)$ & $0.9(0.8,1.1)$ & 0.185 \\
\hline nVI-TMSR & $1.0(0.9,1.2)$ & $1.1(0.9,1.2)$ & 0.43 & $1.1(0.9,1.2)$ & $1.0(0.9,1.2)$ & 0.366 \\
\hline
\end{tabular}

* Significantly different between groups after FDR correction.

Marker of inflammation: hsCRP, soluble CD14, and soluble CD163. Marker of microbial translocation: plasma IFABP. Marker of endothelial activation: plasma vWf. Calibrated TG was triggered with $5 \mathrm{pM}$ tissue factor, $4 \mu \mathrm{M}$ phospholipids, and in the presence and absence of $7 \mathrm{nM}$ TM. Lag time (LagT), TTP, ETP. Normalized Protein C activity (NSR). $P$ values were calculated using the Mann-Whitney $U$ test. Circulating factors and ETP as the primary outcome for TG were corrected for multiple testing using the Benjamini-Hochberg procedure (FDR). All remaining TG parameters were treated as exploratory parameters.

sCD14, and sCD163) and the microbial translocation marker IFABP (data summarized in Table 2). D-dimer was independently associated with age $(\mathrm{B}=7.44, P<0.001)$, and after correction for age and sex, the difference in $\mathrm{D}$-dimer between PLHIV and uninfected controls disappeared $(\mathrm{B}=0.154, P=$ 0.878; see Table 1, Supplemental Digital Content, http://links. lww.com/QAI/B606). The markers of inflammation and microbial translocation remained significantly increased in PLHIV compared with those of controls after correction for age and sex (see Table 1, Supplemental Digital Content, http:/links.lww.com/QAI/B606). D-dimer concentrations correlated with markers of inflammation, IFABP, and endothelial activation (plasma vWF; Fig. 1).

\section{Ex Vivo Coagulation Capacity by TG}

Next, ex vivo coagulation capacity was determined by ex vivo calibrated TG in plasma. Overall, calibrated thrombin generation showed lower TG, most notably reduced ETP, our primary outcome regarding TG, in PLHIV compared with that in uninfected controls (Table 2, FDRcorrected). In addition, PLHIV experienced reduced peak thrombin formation and increased LT compared with HIVnegative controls (data summarized in Table 2). This difference in ETP persisted (FDR-corrected) only in a subgroup analysis of participants aged 40 years and older (see Table 2, Supplemental Digital Content, http://links. lww.com/QAI/B606), but disappeared in the subgroup of only men (see Table 3, Supplemental Digital Content, http:// links.lww.com/QAI/B606). However, after correction for age and sex, none of the TG parameters were significantly different between both cohorts (see Table 1, Supplemental Digital Content, http://links.lww.com/QAI/B606). Most women used oral contraceptive during sampling, which may account for the strong changes in TG parameters when stratifying for sex in the uninfected cohort. ${ }^{29}$ Within the PLHIV cohort, no clear association was found between ETP and CD4 nadir, HIV duration, or current CD4 count (Fig. 1; see Table 5, Supplemental Digital Content, http://links.lww. com/QAI/B606). Higher current CD4 count and CD4 recovery were correlated with increased peak thrombin and ETP (data summarized in Table 5, Supplemental Digital Content, http://links.lww.com/QAI/B606), whereas HIVRNA zenith and HIV duration were associated with decreased protein C activity (nETP-TMsr; see Table 4, Supplemental Digital Content, http://links.lww.com/QAI/ B606). With the exception of nETP-TMsr, we found no associations between smoking and TG parameters (see Table 5, Supplemental Digital Content, http://links.lww.com/QAI/ B606). 


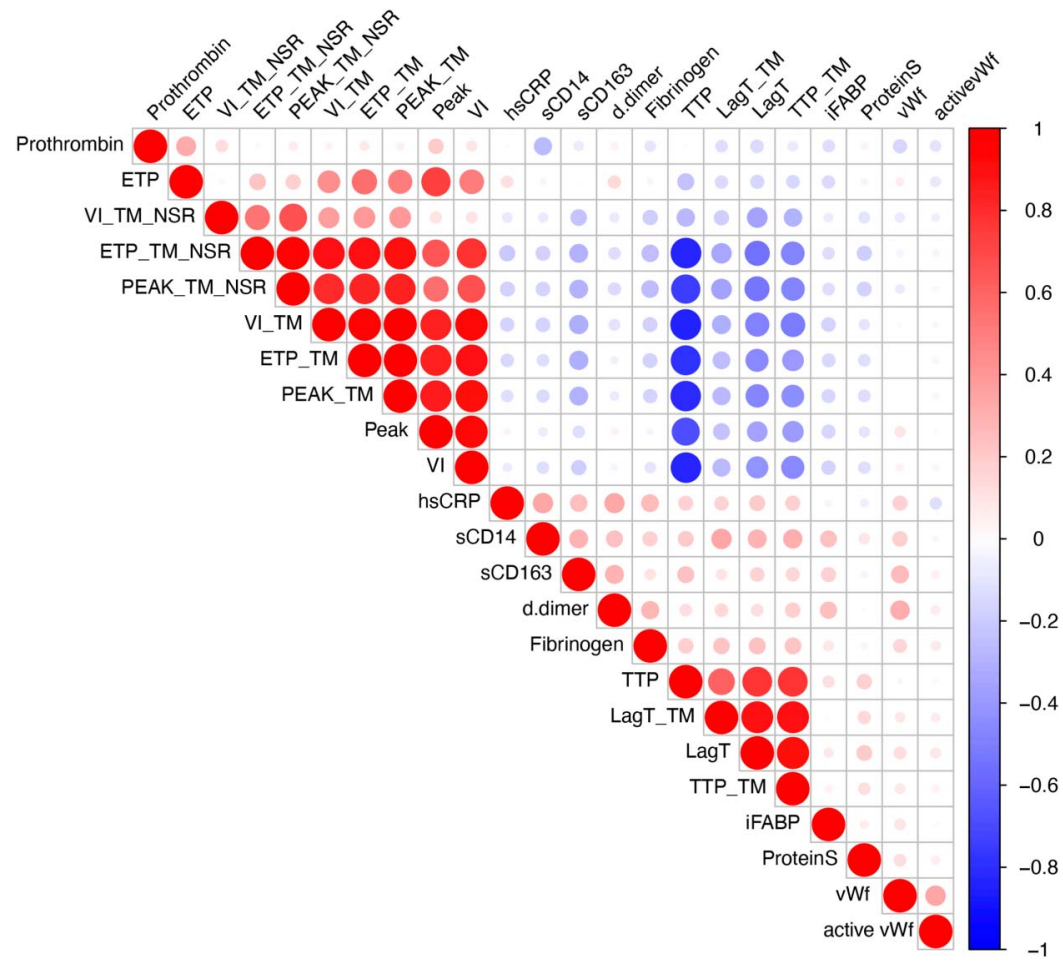

FIGURE 1. Spearman correlation matrix. Spearman correlation coefficients are shown in color and size of circles. Parameters are clustered using hierarchal clustering based on coefficients. Markers of inflammation: hsCRP, soluble CD14, and soluble CD163. Marker of microbial translocation: plasma IFABP. Marker of endothelial activation: plasma vWf. Calibrated TG was triggered with 5 pM tissue factor, $4 \mu \mathrm{M}$ phospholipids, and in the presence and absence of 7 nM TM. Lag time (LagT), TTP, and ETP. Normalized protein C activity (NSR). IFABP, intestinal fatty acid binding protein. full color

\section{Inflammation and Ex Vivo Coagulation Capacity}

Although inflammatory markers and D-dimer correlated positively, ETP did not correlate with any of the included inflammatory markers. Only LT and TTP correlated with sCD14, sCD163, and hsCRP (data shown in Fig. 1). Moreover, sCD163 showed a negative correlation with protein $\mathrm{C}$ activity (nETP-TMsr; $\mathrm{R}-0.2, P<0.05$, Fig. 1 ).

\section{Increased TG in Abacavir- vs Non-abacavir- treated PLHIV}

Next, we explored whether different antiretroviral drugs were associated with TG. There was no association between the use of a protease inhibitor, INSTI, or nonnucleoside reverse transcriptase inhibitor (NNRTI; Fig. 2) with ETP or any of the other TG parameters. By contrast, abacavir use was associated with an increased ETP [non-abacavir: $83.7 \%(74.7,97.6)$ vs $90.2 \%$ (82.7, 101.6), $P=0.001$; Fig. 3 and Table 2]. In addition, there was a trend toward higher peak thrombin concentrations in the abacavir group $[79.9 \%(65.2,96.0)$ vs $85.8 \%(76.1,95.7) P=$ 0.083; Table 2]. This effect of abacavir on ETP was independent of age, sex, and inflammation (ETP: $\mathrm{B}=8.00, P=0.006$; see Table 5, Supplemental Digital Content, http://links.lww.com/QAI/ B606). Usage of tenofovir difumarate (TDF) was the most prescribed alternative for abacavir. As a consequence, TDF was associated with a reduced ETP in our cohort $(\mathrm{B}=-7.86, P=$ -0.006 ). However, in multivariate analysis, the effect of abacavir seemed to be larger than TDF on ETP. Therefore, we attributed the effect of NRTIs on ETP to abacavir in this study. To a similar extent, we could not clearly discriminate between lamivudine and abacavir in this cohort because this was the most prescribed combination of NRTIs (all data from multivariate analyses are tabulated in Table 4, Supplemental Digital Content, http://links. lww.com/QAI/B606). Because INSTI use was high in the abacavir group and showed a trend toward lower ETP, we corrected for INSTI use $(\mathrm{B}=-5.79, P=0.061)$, and the effect of abacavir on ETP became more pronounced (ETP: $\mathrm{B}=8.85, P=0.002$ ).

The abacavir-associated increase in ETP was not due to decreased protein $\mathrm{C}$ activity, a known inhibitor of plasmatic coagulation, because nETPsr was comparable between groups even after correcting for age, sex, and inflammation (abacavir: $\mathrm{B}=-0.895, P=0.37$ ).

In most analyses on abacavir-associated cardiovascular risk in treated PLHIV, there was a discrepancy between current use, past use ( $>6$ months), and cumulative abacavir use (reviewed by Llibre et al. ${ }^{30}$ ). Current use of abacavir was more strongly associated with ETP in our cohort than cumulative use. When analysis was restricted to current abacavir users, cumulative exposure (in days) was not correlated with ETP $(\mathrm{B}=0.597, P=0.552)$.

When restricting the analysis to non-abacavir users, PLHIV had a decreased ETP, with increased lag time and more pronounced protein $\mathrm{C}$ activity (Table 2 and Table 6, Supplemental Digital Content, http://links.lww.com/QAI/ B606), when compared with uninfected controls.In summary, our data show that abacavir use is associated with an increase in ex vivo coagulation capacity reflected by increased TG, whereas overall, PLHIV on a non-abacavir regimen showed a decreased coagulation capacity measured by TG.

\section{Mechanisms of Decreased TG}

Decreased TG and increased D-dimer concentrations suggest consumption of coagulation factors. Indeed, 

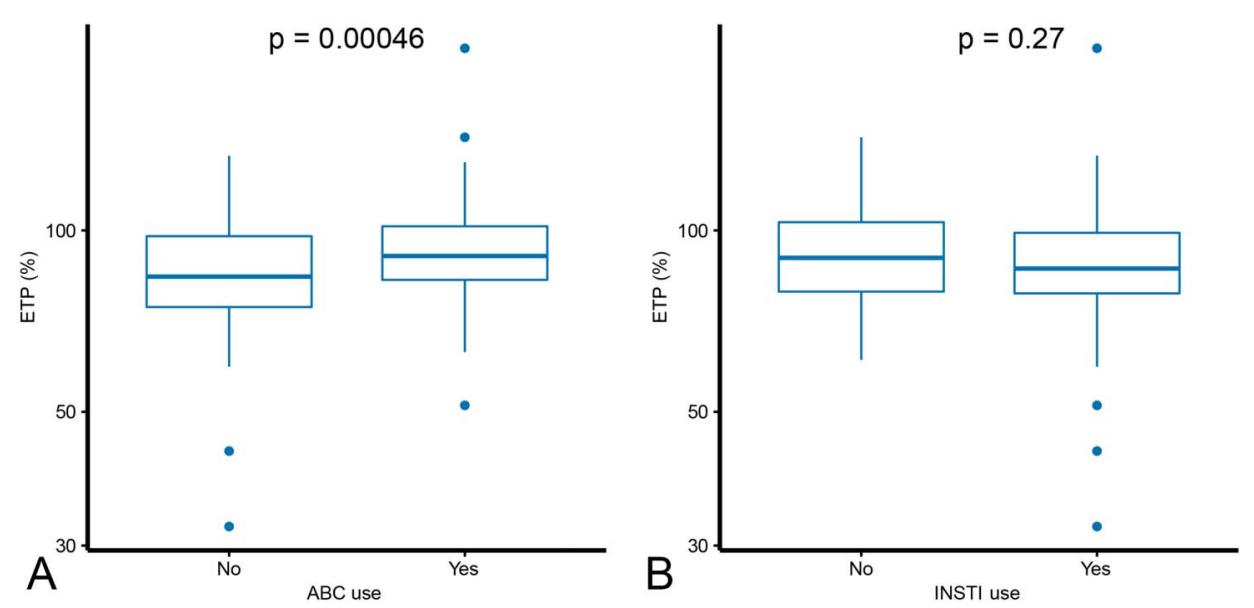

FIGURE 2. Endogenous thrombin potential stratified per CART use. ETP $(\mathrm{nM} \times \mathrm{min})$ were normalized as the percentage of that of NPP tested in the same run. ABC, Abacavir; INSTI, integrase inhibitor; RAL, raltegravir, DTG, dolutegravir. Values were compared using the Mann-Whitney $U$ test. Data are depicted according to the Tukey procedure. Comparisons are corrected for multiple testing using the Benjamini-Hochberg procedure. ETP remained significantly higher in $A B C$ users compared with non-ABC after FDR correction. full color
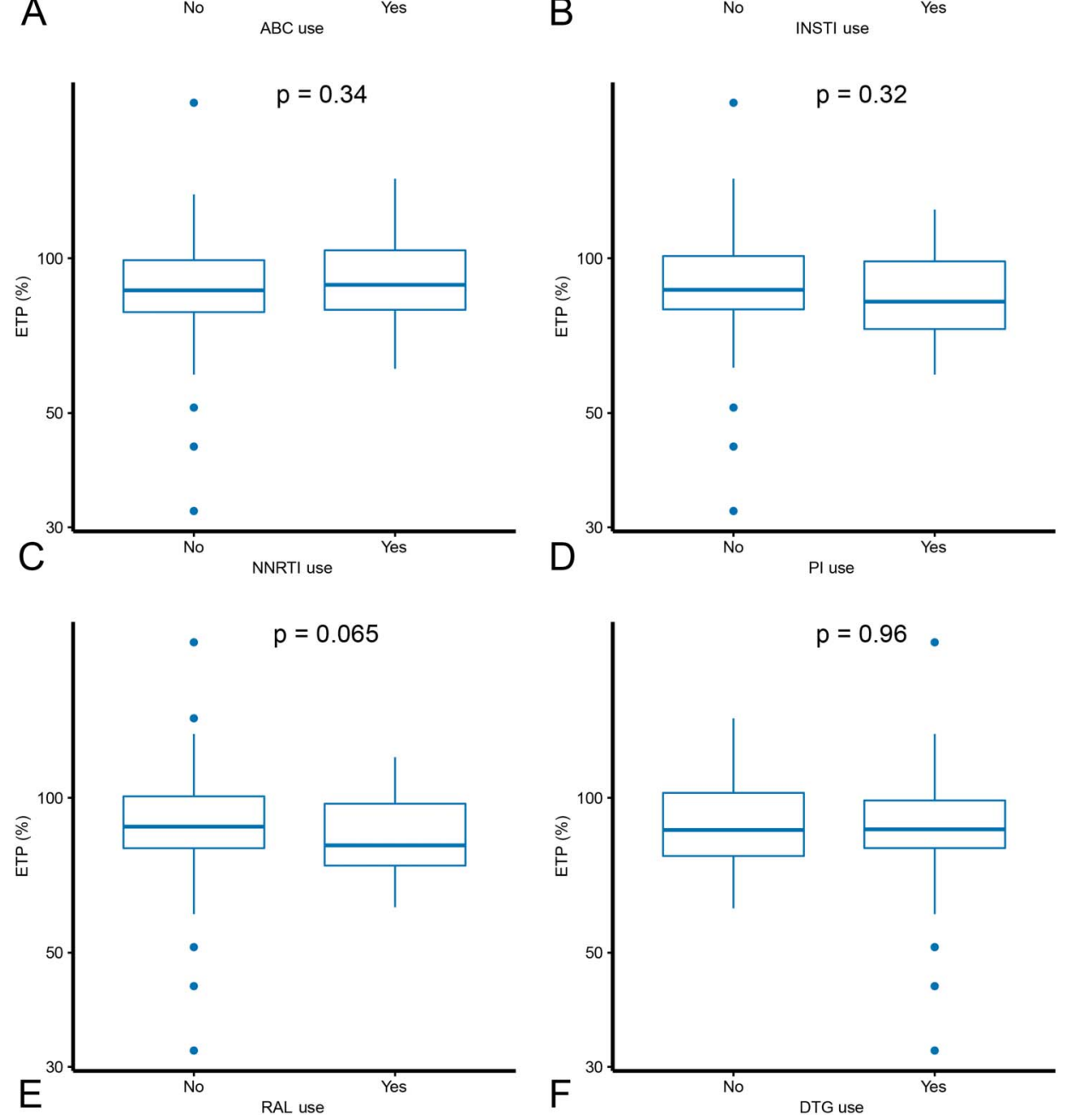

prothrombin concentrations were decreased in PLHIV (Fig. 3C), even after correcting for age and sex [PLHIV: 109.0\% (89.3, 135.1), HC: 135.1\% (102.4, 162.5), $P<0.001$; see Table 1, Supplemental Digital Content, http://inks.lww.com/ QAI/B606]. Although prothrombin concentrations were decreased in PLHIV compared with those in controls, fibrinogen concentrations [PLHIV: $3.4 \mathrm{mg} / \mathrm{mL}(2.8,4.0)$, HC: $3.2 \mathrm{mg} / \mathrm{mL}(2.8,4.1), P=0.746]$ did not differ between groups (Figs. 3A, C). Furthermore, prothrombin was strongly

correlated with ETP parameters in both PLHIV and uninfected controls (Fig. 3D and Table 6, Supplemental Digital Content, http://links.lww.com/QAI/B606), suggesting that the reduced concentration of prothrombin may underlie the observed decrease in TG in PLHIV. Reduced production of prothrombin and other coagulation factors (by the liver) was deemed unlikely because of similar concentrations of protein S [PLHIV: 91.7\% (81.7, 109.4), HC: 86.9\% (72.7, 112.4), $P=0.163$, Fig. 3E] and fibrinogen. No difference in 

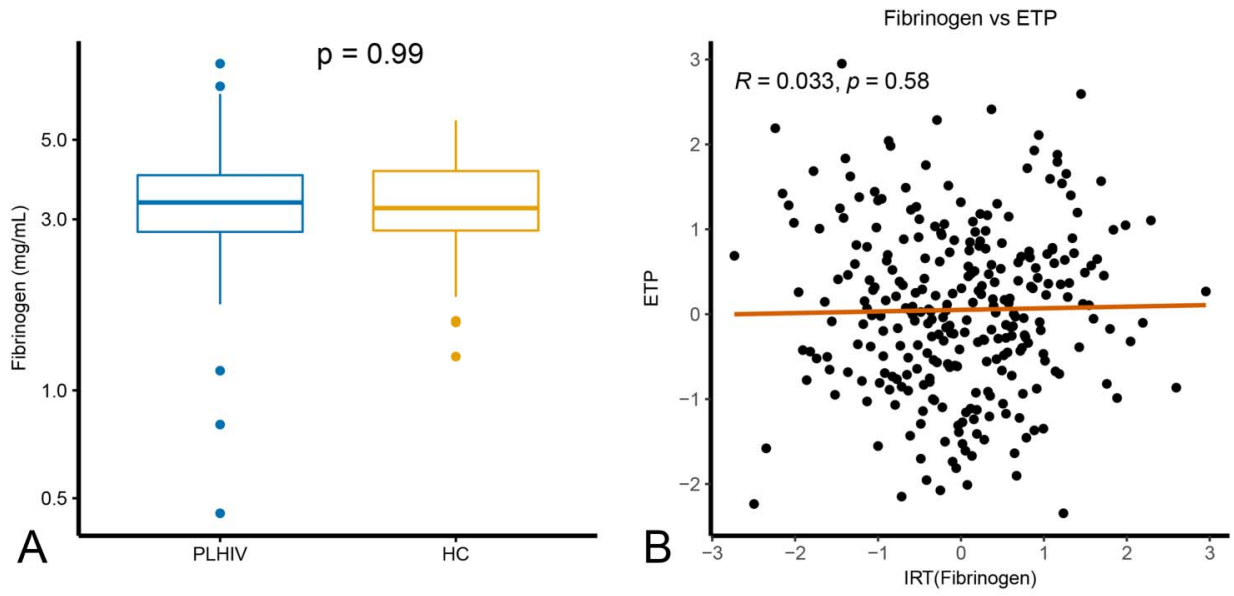

FIGURE 3. Coagulation factors in PLHIV and healthy controls. A, Fibrinogen concentration $(\mathrm{mg} / \mathrm{mL})$ in PLHIV and HC. B, Correlation between inverse rank-based transformed (IRT) ETP (percentage of NPP) and fibrinogen. C, Prothrombin concentrations (percentage of NPP) in PLHIV and controls. D, Correlation plot for IRT prothrombin and IRT ETP. E, Protein S concentrations (\% of NPP) in PLHIV and controls. F, IRT protein $S$ and IRT ETP correlation plot. HC, healthy controls. Correlations coefficients are Pearson coefficients after IRT for normalization.

Comparisons between cohorts were performed using the Mann-Whitney $U$ test. full color
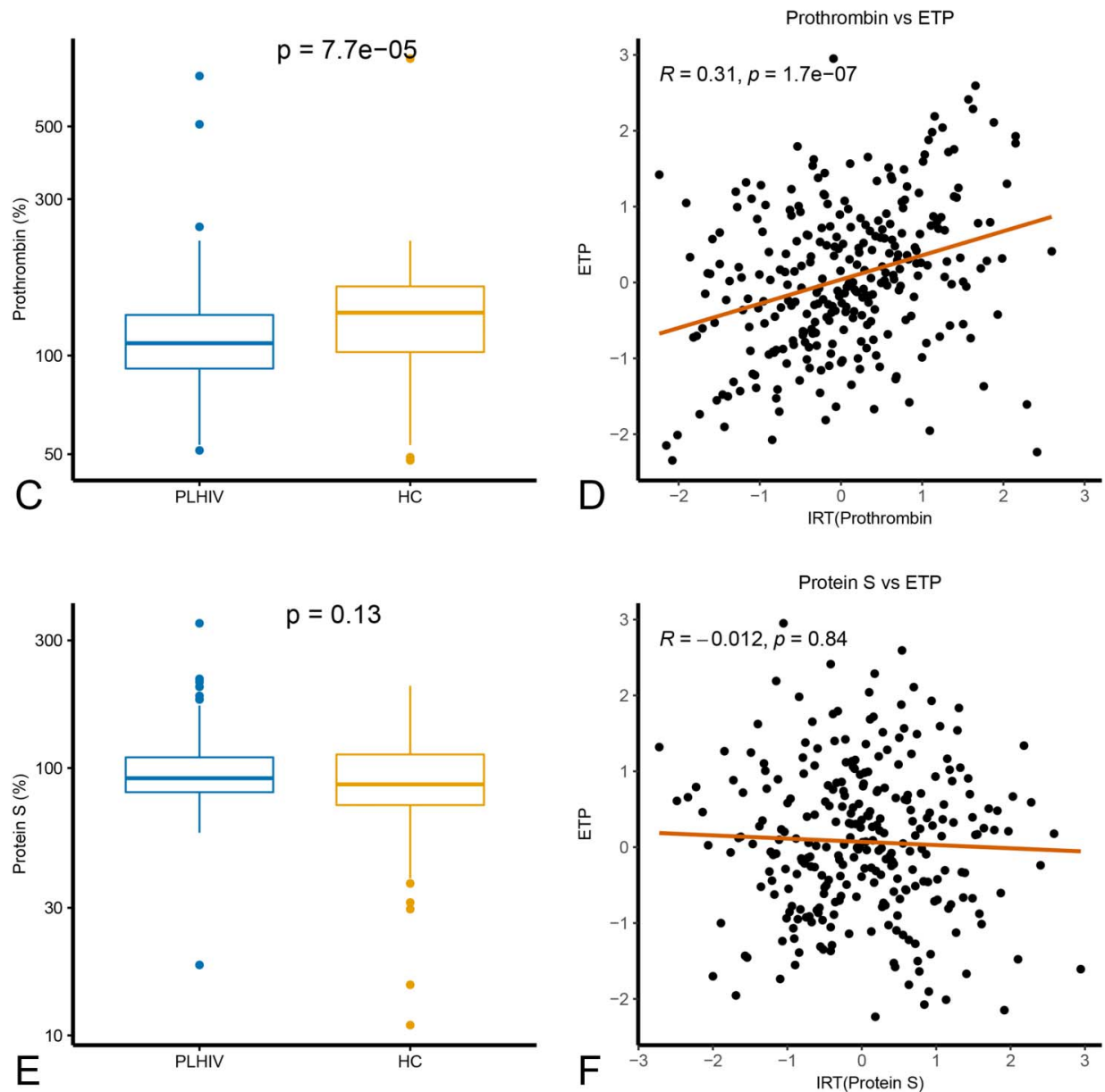

prothrombin or fibrinogen could be observed when PLHIV were stratified based on abacavir use (Table 2).

\section{DISCUSSION}

In a cohort of virally suppressed PLHIV on chronic cART, we show that abacavir use was associated with increased TG compared with that of non-abacavir regimens.
Overall, PLHIV exhibited reduced TG compared withHIVuninfected controls, which was associated with lower plasma concentrations of prothrombin. PLHIV had higher concentrations of inflammatory markers, which correlated with D-dimer concentrations, but not with TG parameters.

TG is a diagnostic tool for hypercoagulability states. This is not limited to venous thrombosis and may also include arterial thrombosis. ${ }^{31}$ The use of abacavir has been linked to an 
increased risk of acute cardiovascular events in different studies, ${ }^{10-13}$ but not in all. ${ }^{14,15}$ By contrast, abacavir use does not seem to be associated with an increased incidence of venous thrombosis. $^{5}$ Increased platelet activation was suggested to underlie the excess cardiovascular risk in users of abacavir, ${ }^{32-34}$ although increased platelet activation was not observed in all studies. $^{35}$ A recent study found that abacavir led to an inflammatory and prothrombotic endothelial phenotype promoting in vivo platelet activation through endothelial microparticles. ${ }^{36}$ The increased TG in abacavir use could also be caused by endothelial dysfunction and possible prothrombotic endothelial microparticles. ${ }^{36,37}$ In addition, thrombin directly activates platelets, so our finding of increased TG capacity in abacavir users may as such contribute to increased platelet activation and CVD. ${ }^{38}$ In contrast to our results, a study by Jong et al. ${ }^{24}$ showed no difference in TG for abacavir use, but only with a limited sample size of 27 individuals in the abacavir group.

Although inflammation was increased in PLHIV, we found no increase in TG. Moreover, the subgroup analysis of non-abacavir users showed a slight decrease in TG in PLHIV compared with that in uninfected controls. This observation is in line with earlier observations, showing decreased TG in longterm-treated PLHIV. ${ }^{25}$ In this study, we confirm these results using a cohort of long-term-treated PLHIV on more recently recommended cART regimens. Regarding possible mechanisms, consumption of clotting factors could play a role because prothrombin concentrations were decreased with a concurrent increase in D-dimer in the total PLHIV group. Fibrinogen concentrations were similar across all groups, but fibrinogen is a well-known acute phase protein and could, therefore, reflect inflammation rather than coagulation capacity. ${ }^{39}$ Furthermore, decreased production of (pro)coagulation factors was deemed less likely because other factors produced by the liver such as fibrinogen and protein $\mathrm{S}$ were similar across groups.

More recent articles have used in silico TG as a proxy for plasmatic coagulation potential and showed that this calculated TG correlates with development of CVD. ${ }^{40}$ Furthermore, in the hallmark randomized controlled SMART trial investigating CD4guided treatment interruption, in silico TG was lower in PLHIV without ART and ongoing viral replication compared with that in cART-treated PLHIV. ${ }^{41,42}$ Although D-dimer mirrors coagulation and fibrinolysis in vivo, in vitro TG reflects actual hemostatic potential of the plasmatic coagulation pathways. ${ }^{22} \mathrm{We}$ confirmed that D-dimers indeed correlated with endothelial activation (eg, vWF) and inflammation (eg, sCD163), but found no such relation with TG parameters in PLHIV. This suggests that increased D-dimer concentrations are primarily influenced by increased provoking signals for coagulation, such as endothelial activation and inflammation during HIV infection. However, increased plasmatic coagulation capacity could still play a role in CVD in PLHIV, as was shown by a case-control study using in silico TG. ${ }^{42}$ A significant role for plasmatic coagulation and increased TG has been shown in clinical studies including uninfected patients at risk for CVD. ${ }^{31}$ Because PLHIV are known for increased inflammation and endothelial activation, an abacavir-associated potentiation of the plasmatic coagulation capacity could, therefore, still result in increased risk for arterial thrombosis.
Interestingly, protein $\mathrm{C}$ activity was higher in PLHIV and correlated with inflammation (sCD163), thereby reducing the overall plasmatic coagulation capacity. This rebalancing of the anticoagulant and procoagulant pathway during HIV infection mimics the rebalancing of hemostasis seen in liver disease. ${ }^{43}$ Monocyte and macrophage activation (sCD14 and sCD163) correlated with LT and TTP. These parameters are mostly influenced by an increase in tissue factor pathway inhibitor (TFPI). ${ }^{44}$ This TFPI is known to be increased in PLHIV. ${ }^{45}$ Although endothelial cells and platelets mainly produce TFPI, monocytes are known producers of TFPI too and could increase production on activation. ${ }^{44}$

\section{LIMITATIONS AND STRENGTHS}

The cross-sectional design of our study does not allow to draw causal inferences. Furthermore, the independent increase in TG seen in abacavir treatment could be affected through indication bias whereby treatment selection is influenced by patient characteristics. Yet, in our case, abacavir was preferably given to people with a decreased cardiovascular risk profile, which was reflected in our cohort with a trend toward lower CVD risk factors in the abacavir-treated group. Second, because TDF was the most prescribed alternative to abacavir, we cannot fully rule out that TDF inhibit TG. Yet, it will not change the conclusions that abacavir is associated with increased TG compared with nonabacavir users. To a similar extent, we cannot exclude the possibility that lamivudine is involved in this difference because lamivudine was prescribed concurrently to abacavir in most patients. Third, we could not correlate TG parameters with clinical outcomes, as was performed in the study reporting on in silico TG. Although in silico TG can be calculated from plasma markers measured in (long-term) stored EDTA plasma, our functional assay of calibrated TG (CAT) requires citrated plasma, which is not regularly collected in large-scale cohorts. Actual measurement of thrombin formation using the CAT method is more precise than the modeling of the in silico TG method because CAT also includes possible unknown confounders such as cART use or other unknown factors. Therefore, CAT better reflects actual hemostatic potential in complex disease states such as an HIV infection. Furthermore, owing to lower number of women in our study, generalizability of our findings to women is limited and warrants further study. Our cohort only consists of virally suppressed PLHIV on long-term treatment and reflects the current HIV-infected population. Thus, our data cannot be used to draw conclusions about patients who are still severely immune compromised or with ongoing viral replication.

In conclusion, abacavir use was associated with increased TG, and this increase could serve as an additional factor in the reported increase in thrombotic events during abacavir use. Increased exposure to triggers that propagate coagulation, such as inflammation, and endothelial activation likely underlie increased D-dimer concentrations found in most PLHIV.

\section{REFERENCES}

1. Knudsen TB, Ertner G, Petersen J, et al. Plasma soluble CD163 level independently predicts all-cause mortality in HIV-1-Infected individuals. J Infect Dis. 2016;214:1198-1204.

2. Baker JV, Neuhaus J, Duprez D, et al. Changes in inflammatory and coagulation biomarkers: a randomized comparison of immediate versus 
deferred antiretroviral therapy in patients with HIV infection. $J$ Acquir Immune Defic Syndr. 2011;56:36-43.

3. Hsu DC, Ma YF, Hur S, et al. Plasma IL-6 levels are independently associated with atherosclerosis and mortality in HIV-infected individuals on suppressive antiretroviral therapy. AIDS. 2016;30:2065-2074.

4. Foley JH, Conway EM. Cross talk pathways between coagulation and inflammation. Circ Res. 2016;118:1392-1408.

5. Howard JFB, Rokx C, Smit C, et al. Incidence of a first venous thrombotic event in people with HIV in The Netherlands: a retrospective cohort study. Lancet HIV. 2019;6:e173-e181.

6. Rasmussen LD, Dybdal M, Gerstoft J, et al. HIV and risk of venous thromboembolism: a Danish nationwide population-based cohort study. HIV Med. 2011;12:202-210.

7. Freiberg MS, Chang CC, Kuller LH, et al. HIV infection and the risk of acute myocardial infarction. JAMA Internc Med. 2013;173:614-622.

8. Armah KA, Chang CC, Baker JV, et al. Prehypertension, hypertension, and the risk of acute myocardial infarction in HIV-infected and -uninfected veterans. Clinical Infect Dis. 2014;58:121-129.

9. Nordell AD, McKenna M, Borges AH, et al. Severity of cardiovascular disease outcomes among patients with HIV is related to markers of inflammation and coagulation. $J$ Am Heart Assoc. 2014;3:e00844.

10. Group DADS, Sabin CA, Worm SW, Weber R, et al. Use of nucleoside reverse transcriptase inhibitors and risk of myocardial infarction in HIVinfected patients enrolled in the D:A:D study: a multi-cohort collaboration. Lancet. 2008;371:1417-1426.

11. Sabin CA, Reiss P, Ryom L, et al. Is there continued evidence for an association between abacavir usage and myocardial infarction risk in individuals with HIV? A cohort collaboration. BMC Med. 2016;14:61.

12. Obel N, Farkas DK, Kronborg G, et al. Abacavir and risk of myocardial infarction in HIV-infected patients on highly active antiretroviral therapy: a population-based nationwide cohort study. HIV Med. 2010;11:130-136.

13. Elion RA, Althoff KN, Zhang J, et al. Recent abacavir use increases risk of type 1 and type 2 myocardial infarctions among adults with HIV. $J$ Acquir Immune Defic Syndr. 2018;78:62-72.

14. Ding X, Andraca-Carrera E, Cooper C, et al. No association of abacavir use with myocardial infarction: findings of an FDA meta-analysis. $J$ Acquir Immune Defic Syndr. 2012;61:441-447.

15. Lang $\mathrm{S}$, Mary-Krause $\mathrm{M}$, Cotte $\mathrm{L}$, et al. Impact of individual antiretroviral drugs on the risk of myocardial infarction in human immunodeficiency virus-infected patients: a case-control study nested within the French Hospital database on HIV ANRS cohort CO4. Arch Intern Med. 2010;170:1228-1238.

16. Baker JV, Sharma S, Grund B, et al. Systemic inflammation, coagulation, and clinical risk in the START trial. Open Forum Infect Dis. 2017;4:ofx262.

17. Duprez DA, Neuhaus J, Kuller LH, et al. Inflammation, coagulation and cardiovascular disease in HIV-infected individuals. PLoS One. 2012;7:e44454.

18. Haugaard AK, Lund TT, Birch C, et al. Discrepant coagulation profile in HIV infection: elevated D-dimer but impaired platelet aggregation and clot initiation. AIDS. 2013;27:2749-2758.

19. Angelidou K, Hunt PW, Landay AL, et al. Changes in inflammation but not in T-cell activation precede non-AIDS-defining events in a casecontrol study of patients on long-term antiretroviral therapy. J Infect Dis. 2018;218:239-248.

20. Funderburg NT, Mayne E, Sieg SF, et al. Increased tissue factor expression on circulating monocytes in chronic HIV infection: relationship to in vivo coagulation and immune activation. Blood. 2010;115:161-167.

21. Borissoff JI, Spronk HM, Heeneman S, et al. Is thrombin a key player in the 'coagulation-atherogenesis' maze? Cardiovasc Res. 2009;82:392-403.

22. Hemker HC, Giesen P, Al Dieri R, et al. Calibrated automated thrombin generation measurement in clotting plasma. Pathophysiol Haemost Thromb. 2003;33:4-15.

23. Jong E, Louw S, Meijers JC, et al. The hemostatic balance in HIVinfected patients with and without antiretroviral therapy: partial restora- tion with antiretroviral therapy. AIDS Patient Care STDS. 2009;23: 1001-1007.

24. Jong E, Meijers JC, van Gorp EC, et al. Markers of inflammation and coagulation indicate a prothrombotic state in HIV-infected patients with long-term use of antiretroviral therapy with or without abacavir. AIDS Res Ther. 2010;7:9.

25. Hsue PY, Scherzer R, Grunfeld C, et al. HIV infection is associated with decreased thrombin generation. Clin Infect Dis. 2012;54:1196-1203.

26. Bloemen S, Kelchtermans H, Hemker HC. Thrombin generation in low plasma volumes. Thromb J. 2018;16:10.

27. Bloemen S, Zwaveling S, Douxfils J, et al. The anticoagulant effect of dabigatran is reflected in the lag time and time-to-peak, but not in the endogenous thrombin potential or peak, of thrombin generation. Thromb Res. 2018;171:160-166.

28. van der Vorm LN, Li L, Huskens D, et al. Analytical characterization and reference interval of an enzyme-linked immunosorbent assay for active von Willebrand factor. PLoS One. 2019;14:e0211961.

29. Burchall GF, Piva TJ, Ranasinha S, et al. Differential effects on haemostatic markers by metformin and the contraceptive pill: a randomized comparative trial in PCOS. Thromb Haemost. 2017;117:2053-2062.

30. Llibre JM, Hill A. Abacavir and cardiovascular disease: a critical look at the data. Antivir Res. 2016;132:116-121.

31. Ten Cate H, Hemker HC. Thrombin generation and atherothrombosis: what does the evidence indicate? J Am Heart Assoc. 2016;5:10.

32. Satchell CS, O'Halloran JA, Cotter AG, et al. Increased platelet reactivity in HIV-1-infected patients receiving abacavir-containing antiretroviral therapy. J Infect Dis. 2011;204:1202-1210.

33. Baum PD, Sullam PM, Stoddart CA, et al. Abacavir increases platelet reactivity via competitive inhibition of soluble guanylyl cyclase. AIDS 2011;25:2243-2248.

34. Taylor KA, Smyth E, Rauzi F, et al. Pharmacological impact of antiretroviral therapy on platelet function to investigate human immunodeficiency virusassociated cardiovascular risk. Br J Pharmacol. 2019;176:879-889.

35. Diallo YL, Ollivier V, Joly V, et al. Abacavir has no prothrombotic effect on platelets in vitro. J Antimicrob Chemother. 2016;71:3506-3509.

36. Khawaja AA, Taylor KA, Lovell AO, et al. HIV antivirals affect endothelial activation and endothelial-platelet crosstalk. Circ Res. 2020; 127:1365-1380.

37. Yau JW, Teoh H, Verma S. Endothelial cell control of thrombosis. BMC Cardiovasc Disord. 2015;15:130.

38. Panova-Noeva M, Neu MA, Eckerle S, et al. Cardiovascular risk factors are important determinants of platelet-dependent thrombin generation in adult survivors of childhood cancer. Clin Res Cardiol. 2019;108:438-447.

39. Davalos D, Akassoglou K. Fibrinogen as a key regulator of inflammation in disease. Semin Immunopathol. 2012;34:43-62.

40. Brummel-Ziedins K, Undas A, Orfeo T, et al. Thrombin generation in acute coronary syndrome and stable coronary artery disease: dependence on plasma factor composition. J Thromb Haemost. 2008;6:104-110.

41. Baker JV, Brummel-Ziedins K, Neuhaus J, et al. HIV replication alters the composition of extrinsic pathway coagulation factors and increases thrombin generation. $J$ Am Heart Assoc. 2013;2:e000264.

42. Brummel-Ziedins KE, Gissel M, Neuhaus J, et al. In silico thrombin generation: plasma composition imbalance and mortality in human immunodeficiency virus. Res Pract Thromb Haemost. 2018;2:708-717.

43. O'Leary JG, Greenberg CS, Patton HM, et al. AGA clinical practice update: coagulation in cirrhosis. Gastroenterology. 2019;157:34-43.e31.

44. Wood JP, Ellery PE, Maroney SA, et al. Biology of tissue factor pathway inhibitor. Blood. 2014;123:2934-2943.

45. Barska K, Kwiatkowska W, Knysz B, et al. The role of the tissue factor and its inhibitor in the development of subclinical atherosclerosis in people living with HIV. PLoS One. 2017;12:e181533. 